\title{
Comparing the EQ-5D 3L and 5L: measurement properties and association with chronic conditions and multimorbidity in the general population
}

Calypse B Agborsangaya ${ }^{1}$, Markus Lahtinen², Tim Cooke² and Jeffrey A Johnson ${ }^{1 *}$

\begin{abstract}
Background: Studies comparing the measurement properties of EQ-5D 3L (3L) and EQ-5D 5L (5L) are limited to specific patient populations with small sample sizes. Using a general population sample, we compared $3 \mathrm{~L}$ and $5 \mathrm{~L}$ in terms of their measurement properties and association with number of chronic conditions, including multimorbidity - the concurrent occurrence of two or more chronic conditions.

Methods: Data were available from two consecutive cycles of a cross-sectional telephone interview survey using $3 \mathrm{~L}$ (2010 cycle) and 5L (2012 cycle), in the general population of adults (age $\geq 18$ years) in Alberta, Canada. Measurement properties were compared by determining their feasibility, ceiling effect, and discriminatory power (Shannon indices) for $3 \mathrm{~L}$ and $5 \mathrm{~L}$. Linear regression models were fitted to test the associations between multimorbidity and EQ-5D index score.

Results: Data were available for 4946 (2010) and 4752 (2012) survey respondents with information on HRQL. Compared to $3 \mathrm{~L}$, $5 \mathrm{~L}$ showed lower ceiling effect (32.3\% versus $42.1 \%)$, higher absolute discriminatory power (Shannon index, mean 0.79 versus 0.52 ) and higher relative discriminatory power (Shannon Evenness index, mean 0.09 versus 0.06 for 3L). Despite these differences, similar relationships of lower HRQL with greater multimorbidity were observed for the $3 \mathrm{~L}$ $(\beta=-0.13,95 \% \mathrm{Cl}-0.15 ;-0.11)$ and $5 \mathrm{~L}(B=-0.12,95 \% \mathrm{Cl}-0.13 ;-0.11)$.

Conclusions: Using a general population sample, the EQ-5D 5L showed better measurement properties than the EQ-5D 3L. Nonetheless, clinically important differences in HRQL associated with multimorbidity were similar in magnitude using both versions of EQ-5D.
\end{abstract}

Keywords: EQ-5D, Quality of life, Multimorbidity, Health related quality of life, Chronic diseases, Shannon index, Canada

\section{Background}

For over two decades, EuroQol's EQ-5D has been used as a generic instrument to measure and evaluate health status [1-3]. The older version, the EQ-5D 3L (3L), describes general health based on five distinct dimensions: mobility, self-care, usual activities, pain/discomfort and anxiety/depression. Each dimension has 3 Levels (indicating no problem, some or moderate problem and extreme

\footnotetext{
* Correspondence: jeff.johnson@ualberta.ca

'Department of Public Health Sciences, 2-040 Li Ka Shing Center for Health Research and Innovation, University of Alberta, Edmonton, Alberta, T6G 2E1, Canada

Full list of author information is available at the end of the article
}

problem). Due to its limited ability to delineate minor but important clinical differences in health status as well as the presence of a ceiling effect, the EuroQol group recently developed a new version, the EQ-5D 5L (5L) [4-7]. The $5 \mathrm{~L}$ version differs from $3 \mathrm{~L}$ in that for each dimension, there are 5 levels (no problem, slight problem, moderate problem, severe problem and extreme problem).

Previous research comparing the $3 \mathrm{~L}$ and $5 \mathrm{~L}$ versions of the EQ-5D indicate that additional levels of the $5 \mathrm{~L}$ potentially increase discriminatory power and reduce ceiling effect among patients with chronic conditions, including chronic hepatic disease [8] or cancer patients 
[9]. Although few studies have compared 3L and 5L, most of them are based on patient populations or studies with relatively small sample sizes [6-9]. In one study among eighty-two participants [6], the authors compared $3 \mathrm{~L}$ and $5 \mathrm{~L}$ and noted a significant improvement in the discriminatory power of the $5 \mathrm{~L}$. More research is needed to obtain a comparative assessment of both versions of the EQ-5D in a larger sample and their applicability in the general population.

The present study aims to compare the measurement properties of 3L and 5L in terms of their feasibility, ceiling effect and discriminatory power (Shannon indices) in a general population sample. In a further step, we compared the association between multimorbidity, the concurrent occurrence of 2 or more chronic conditions, and health-related quality of life (HRQL) using both versions of the EQ-5D.

\section{Methods}

\section{Study setting and population}

The study sample constitutes respondents to two consecutive survey cycles of the Health Quality Council of Alberta (HQCA) Patient Experience and Satisfaction Survey [10] for 2010 and 2012. In this cross-sectional survey, adult Albertans aged 18 years or older, representative of the adult general population, self-reported their experiences and satisfaction with the quality of health services received in the past twelve months. The survey comprised of a telephone-based questionnaire that was administered by Random-Digit Dialing (RDD). In the sampling design, densely populated regions were undersampled and sparsely populated regions were oversampled to ensure a reasonably large sample for analysis and reporting of data for each health region. This study uses combined regional samples to represent the population of Alberta. In doing so, sampling weights were applied to adjust for under- and over-sampling.

\section{Chronic conditions and socio-demographic factors}

Respondents were asked about their health status in the past 12 months, including if they had "any of the following chronic conditions"; diabetes, chronic obstructive pulmonary disorder, asthma, hypertension, high cholesterol, sleep apnea, congestive heart failure, depression or anxiety, chronic pain, arthritis, heart disease, stroke (or related), cancer, gastro-intestinal tract and kidney diseases. Thus, this analysis includes up to fifteen chronic conditions. Respondents were also categorized as having multimorbidity if they had any two or more of these conditions. The survey respondents also gave information on their socio-demographic characteristics such as age, sex, educational attainment and household income. Unlike in 2012, the 2010 survey cycle included a skip pattern for listing chronic conditions that queried respondents to indicate if they have "any chronic condition" prior to the list of chronic conditions.

\section{Measures}

The 2010 survey cycle included EQ-5D 3L and has been described elsewhere [3,11], whereas the 2012 cycle entailed the newer version, EQ-5D 5L. Considering that the descriptive system of the EQ-5D comprises of five domains, each with three possible levels for 3L and five levels for $5 \mathrm{~L}$, a combination of the characteristic levels produces $243\left(3^{5}\right)$ possible health states for 3L (ranging from 11111 to 33333$)$ and $3125\left(5^{5}\right)$ health states for 5L (ranging from 11111 to 55555). 11111 represents the best possible health state whereas 33333 (for 3L) or 55555 (for $5 \mathrm{~L}$ ) represent the worst health states.

The EQ-5D index scores are utilities derived from the respondents profile and ranges from 0 to $1 ; 0$ meaning death and 1 complete health [1]. Values less than 0 indicate health states worse than death. Since the EQ-5D index score is a weighted summary score of five items representing different dimensions of health, changes in the EQ-5D index score may arise from different patterns of impairment across individual dimensions. We were particularly interested in (a) the frequency of reported problems in the $3 \mathrm{~L}$ and $5 \mathrm{~L}$ and (b) their EQ-5D single index score. The EQ-5D index scores from 5L were derived as described elsewhere [12,13], using a Crosswalk Index Value Calculator based on US national scoring algorithms. A difference of 0.03 (3\%) was considered to be clinically important [14]. Given that index scores for $5 \mathrm{~L}$ are generated by mapping responses on to $3 \mathrm{~L}$, we hypothesize that significant differences in association will not be observed.

\section{Data analyses}

\section{Discriminatory power}

Shannon index $\left(\mathrm{H}^{\prime}\right)$ and Shannon Evenness index $\left(\mathrm{J}^{\prime}\right)$ were calculated for both instruments as a measure of the discriminatory power. Shannon indices originate from the field of information theory and are typically used to measure species diversity and richness in ecological studies or information richness in the information sciences [15-17]. Some studies have shown that Shannon indices are useful in assessing the discriminatory power in the dimensions of the EQ-5D descriptive system $[4,7,18,19]$. H' is calculated based on the following equation:

$$
\mathrm{H}^{\prime}=-\sum_{i=1}^{L} p_{i} \log _{2} p_{i}
$$

Where L stands for the total number of possible categories (levels), $\mathrm{p}_{\mathrm{i}}=\mathrm{n}_{\mathrm{i}} / \mathrm{N}$, the proportion of recorded observations in the ith level, $\mathrm{n}_{\mathrm{i}}$ the observed number of 
responses in level $i$ and $N$, the total sample size [15]. A higher $\mathrm{H}^{\prime}$ is attributed to more information that has been captured. To calculate $\mathrm{H}^{\prime}$ for an instrument $\left(\mathrm{H}^{\prime}\right.$ instrument $)$, $\mathrm{p}_{\mathrm{i}}$ is generated for the total number of responses for a given level across all dimensions as a proportion of total responses to the entire instrument [4]. Similarly, a higher $\mathrm{H}^{\prime}$ instrument indicates that more information is captured by that instrument. Shannon Evenness Index, J', is a complementary index that reflects the evenness of a distribution and is defined as: $\mathrm{J}^{\prime}=\mathrm{H}^{\prime} / \mathrm{H}^{\prime}{ }_{\max }$, where:

$$
\mathrm{H}_{\text {max }}^{\prime}=\log _{2} \mathrm{~L}
$$

Both indices are descriptive measures of the discriminatory ability of an instrument and are needed for useful interpretation of the measurement power of a scale. We expect that the $\mathrm{H}^{\prime}$ for $5 \mathrm{~L}$ should be higher than for $3 \mathrm{~L}$, and that J' should be about the same or slightly higher for $5 \mathrm{~L}$, indicating the usefulness of the extra levels in the $5 \mathrm{~L}$.

\section{Feasibility}

We tested feasibility by computing the percentage of respondents with missing responses. That is, we calculated the percentage of respondents not answering each dimension. The percentages were then compared for both the $3 \mathrm{~L}$ and $5 \mathrm{~L}$.

\section{Ceiling effect}

The ceiling of $3 \mathrm{~L}$ and $5 \mathrm{~L}$ were defined as the proportion of respondents that scored no problem on all five dimensions (persons with 11111 score). Based on the assumption that the majority of respondents would report at least slight/some problem on at least one of the five dimensions, we hypothesis that the ceiling effect will be lower for $5 \mathrm{~L}$ compared to $3 \mathrm{~L}$ version.

\section{Statistical methods}

Descriptive statistics were performed to determine the sample characteristics for each survey cycle. We presented the proportion of reported problems in all five dimensions for each cycle (without merging) to provide information on the advantage of the extra levels in each dimension. Multivariable linear regression models were then fitted to study associations between chronic conditions and EQ-5D index scores. Three separate models were fitted for 1) specific chronic conditions, 2) for number of chronic conditions and 3) for presence of multimorbidity as a binary categorical variable. The multivariable models were adjusted for respondents' socio-demographic characteristics such as age, sex, and household income. Because educational level tends to correlate with income, we determined a priori not to adjust for educational level to avoid multicollinearity. A 2-sided $\mathrm{P}<0.05$ was considered statistically significant. All analyses were undertaken using STATA version 11 (StataCorp LP. 2009). The Health Research Ethics Board (HREB) at the University of Alberta approved the data collection protocols and survey instruments.

\section{Results}

Socio-demographic characteristics of respondents

4946 (98.7\%) and 4752 (98.9\%) respondents reported data on the EQ-5D in 2010 and 2012 survey cycles, respectively. Respondents' socio-demographic characteristics were comparable in both surveys (Table 1). 52.3\% were female and the mean age was 46.6 (16.5) years for the 2010 cycle, compared to $55.7 \%$ female and mean age of 47.7 (SD, 17.1) years for the 2012 survey. The proportions of respondents reporting five or more chronic conditions were 159 (3.2\%) and 301 (6.3\%) for the 2010 and 2012 survey cycles, respectively. The mean

Table 1 Socio-demographic characteristics of the survey respondents

\begin{tabular}{|c|c|c|}
\hline & $\begin{array}{c}2010 \text { Survey } \\
(\mathrm{N}=4946)\end{array}$ & $\begin{array}{l}2012 \text { Survey } \\
(\mathrm{N}=4752)\end{array}$ \\
\hline Mean age (SD), years & $46.6(16.5)$ years & 47.7 (17.1) years \\
\hline \multicolumn{3}{|l|}{ Sex } \\
\hline Females (\%) & $2585(52.3)$ & $2647(55.7)$ \\
\hline \multicolumn{3}{|l|}{ Age (\%) } \\
\hline $18-28$ & $753(15.2)$ & $724(15.2)$ \\
\hline $29-38$ & $960(19.4)$ & $884(18.6)$ \\
\hline $39-48$ & $1035(20.9)$ & $797(16.8)$ \\
\hline $49-58$ & $987(20.0)$ & $971(20.4)$ \\
\hline $59-68$ & $667(13.5)$ & 799 (16.8) \\
\hline$\geq 69$ & $544(11.0)$ & $577(12.1)$ \\
\hline \multicolumn{3}{|l|}{ Education (\%) } \\
\hline Secondary or less & $1747(35.5)$ & $1634(34.5)$ \\
\hline Post-secondary/College & $2008(40.8)$ & $1896(40.0)$ \\
\hline University degree & $1170(23.8)$ & $1207(25.5)$ \\
\hline \multicolumn{3}{|c|}{ Household income (CAD), \% } \\
\hline$<30,000$ & $579(13.5)$ & $624(15.1)$ \\
\hline $30,000-59,999$ & $1019(23.8)$ & $926(22.4)$ \\
\hline $60,000-99,999$ & $1230(28.7)$ & $1097(26.5)$ \\
\hline$\geq 100,000$ & $1454(34.0)$ & $1496(36.1)$ \\
\hline \multicolumn{3}{|c|}{ Number of chronic conditions } \\
\hline 0 & $3281(66.7)$ & $2277(47.9)$ \\
\hline 1 & $751(15.3)$ & $878(18.5)$ \\
\hline 2 & $387(7.9)$ & $644(13.6)$ \\
\hline 3 & $210(4.3)$ & $388(8.2)$ \\
\hline 4 & $134(2.7)$ & $264(5.6)$ \\
\hline $5+$ & $159(3.2)$ & $301(6.3)$ \\
\hline
\end{tabular}


number of chronic conditions was $0.7(\mathrm{SD}=1.4)$ for the 2010 survey cycle and $1.3(\mathrm{SD}=1.7)$ for the 2012 survey cycle.

\section{EQ-5D profile and morbidity status}

The HRQL profile of persons with multimorbidity is summarized in Table 2. Among persons with multimorbidity, the proportion of respondents reporting slight problems was higher for each dimension of 3L compared to $5 \mathrm{~L}$. For instance, the proportion of persons reporting slight problems with mobility was $45.2 \%$ for $3 \mathrm{~L}$ compared to $25.0 \%$ for $5 \mathrm{~L}$. The same was true for usual activity (69\% for $3 \mathrm{~L}$ versus $23.0 \%$ for $5 \mathrm{~L}$ ). Pain/discomfort appeared to be most common among persons with multimorbidity.
Specific chronic conditions and EQ-5D index score

The mean (SD) index score was 0.87 (SD 0.15) for $3 \mathrm{~L}$ and 0.86 (SD, 0.14) for $5 \mathrm{~L}$. The associations between specific chronic conditions and HRQL are presented in Table 3. In the multivariate analysis, all chronic conditions were associated with clinically important differences in EQ-5D index score using both $3 \mathrm{~L}$ and $5 \mathrm{~L}$. Overall, the associations tended to be similar, albeit, coefficients were larger for $3 \mathrm{~L}$ compared to $5 \mathrm{~L}$ for anxiety or depression, chronic pain, arthritis and sleep apnea (Table 3), in some cases larger than the minimally important difference.

\section{Multimorbidity and EQ-5D index score}

The associations between number of chronic conditions, including multimorbidity, and EQ-5D index score is

Table 2 EQ-5D profile ( $3 \mathrm{~L}$ and $5 \mathrm{~L}$ ) of the survey respondents with multimorbidity

\begin{tabular}{|c|c|c|c|c|c|}
\hline \multicolumn{3}{|l|}{ Multimorbidity (3L) } & \multicolumn{3}{|c|}{ Multimorbidity (5L) } \\
\hline Dimension & $\mathbf{n}$ & $\%$ & $\mathbf{n}$ & $\%$ & Dimensions \\
\hline Mobility & & & & & Mobility \\
\hline No problems & 487 & 54.0 & 823 & 50.7 & No problems \\
\hline Slight problems & 408 & 45.2 & 406 & 25.0 & Slight problems \\
\hline \multirow[t]{3}{*}{ Extreme problems } & 7 & 0.8 & 276 & 17.0 & Moderate problems \\
\hline & & & 104 & 6.4 & Severe problems \\
\hline & & & 13 & 0.8 & Unable to walk about \\
\hline Selfcare & & & & & Selfcare \\
\hline No problems & 813 & 89.7 & 1438 & 88.4 & No problems \\
\hline Slight problems & 91 & 10.0 & 113 & 7.0 & Slight problems \\
\hline \multirow[t]{3}{*}{ Extreme problems } & 2 & 0.2 & 60 & 3.7 & Moderate problems \\
\hline & & & 9 & 0.6 & Severe problems \\
\hline & & & 6 & 0.4 & Unable to wash or dress \\
\hline Usual activities & & & & & Usual activities \\
\hline No problems & 498 & 55.0 & 868 & 53.6 & No problems \\
\hline Slight problems & 380 & 41.9 & 372 & 23.0 & Slight problems \\
\hline \multirow[t]{3}{*}{ Extreme problems } & 28 & 3.1 & 269 & 16.6 & Moderate problems \\
\hline & & & 82 & 5.1 & Severe problems \\
\hline & & & 28 & 1.7 & Unable to do usual activity \\
\hline Pain/Discomfort & & & & & Pain/Discomfort \\
\hline No problems & 170 & 18.8 & 280 & 17.3 & No problems \\
\hline Slight problems & 627 & 69.2 & 586 & 36.2 & Slight problems \\
\hline \multirow[t]{3}{*}{ Extreme problems } & 109 & 12.0 & 535 & 33.1 & Moderate problems \\
\hline & & & 174 & 10.8 & Severe problems \\
\hline & & & 43 & 2.7 & Extreme pain \\
\hline Anxiety/Depression & & & & & Anxiety/Depression \\
\hline No problems & 534 & 59.5 & 868 & 53.8 & No problems \\
\hline Slight problems & 314 & 35.0 & 391 & 24.2 & Slight problems \\
\hline \multirow[t]{3}{*}{ Extreme problems } & 49 & 5.5 & 260 & 16.1 & Moderate problems \\
\hline & & & 66 & 4.1 & Severe problems \\
\hline & & & 29 & 1.9 & Extreme problems \\
\hline
\end{tabular}




\begin{tabular}{lcc}
$\begin{array}{l}\text { Table } 3 \text { Associations between specific chronic conditions } \\
\text { and EQ index score }\end{array}$ & \\
\hline Chronic conditions & $\begin{array}{c}3 \text { L index score } \\
\text { Coef. }(95 \% \text { Cl) }\end{array}$ & $\begin{array}{c}\text { 5 L index score } \\
\text { Coef. }(95 \% \text { Cl})^{2}\end{array}$ \\
\hline Diabetes & $-0.05(-0.08,-0.02)$ & $-0.04(-0.06,-0.02)$ \\
COPD & $-0.10(-0.16,-0.04)$ & $-0.08(-0.11,-0.05)$ \\
Asthma & $-0.06(-0.09,-0.03)$ & $-0.06(-0.07,-0.04)$ \\
High blood pressure & $-0.06(-0.08,-0.04)$ & $-0.06(-0.07,-0.04)$ \\
High cholesterol & $-0.05(-0.08,-0.03)$ & $-0.04(-0.06,-0.03)$ \\
Sleep apnea & $-0.13(-0.17,-0.09)$ & $-0.09(-0.11,-0.06)$ \\
Congestive heart failure & $-0.12(-0.21,-0.03)$ & $-0.12(-0.19,-0.05)$ \\
Depression or anxiety & $-0.19(-0.21,-0.16)$ & $-0.14(-0.15,-0.13)$ \\
Chronic pain & $-0.19(-0.21,-0.17)$ & $-0.17(-0.18,-0.15)$ \\
Arthritis & $-0.12(-0.14,-0.10)$ & $-0.10(-0.12,-0.09)$ \\
Heart disease & $-0.06(-0.10,-0.02)$ & $-0.06(-0.09,-0.03)$ \\
Stroke (or related) & $-0.11(-0.19,-0.02)$ & $-0.12(-0.17,-0.06)$ \\
Cancer & $-0.06(-0.11,-0.01)$ & $-0.05(-0.08,-0.02)$ \\
Kidney disease & $-0.07(-0.20,0.05)$ & $-0.14(-0.19,-0.09)$ \\
Bowel disorders & $-0.09(-0.13,-0.06)$ & $-0.10(-0.13,-0.08)$ \\
\hline
\end{tabular}

${ }^{1}$ EQ-5D 3L from 2010 Survey and ${ }^{2} E Q-5 D$ 5L from 2012 surveys, adjusted for age, sex and household income.

presented in Table 4 . In the multivariable analysis, having a chronic condition was associated with clinically important differences in EQ-5D index score using both $3 \mathrm{~L}$ and $5 \mathrm{~L}$. The difference in EQ-5D index score tended to increase with increasing number of chronic conditions, being highest for persons with five or more conditions $((\beta=-0.23(95 \% \mathrm{CI}-0.27,-0.19)$ for $3 \mathrm{~L}$ and $(ß=-0.22(95 \% \mathrm{CI}-0.23,-0.20)$ for $5 \mathrm{~L})$. Multimorbidity was associated with clinically important differences in EQ-5D index score $\{(\beta=-0.13(95 \% \mathrm{CI}-0.15,-0.11)$ for $3 \mathrm{~L}$ and $\beta=-0.12(95 \% \mathrm{CI}-0.13,-0.11)$ for $5 \mathrm{~L})$.

$\begin{aligned} & \text { Table } 4 \text { Associations between multimorbidity and EQ } \\
& \text { index score }\end{aligned}$
\begin{tabular}{lcc}
$\begin{array}{l}\text { Number of chronic } \\
\text { conditions }\end{array}$ & $\begin{array}{c}\text { EQ-5D } 3 \mathrm{~L} \text { index score } \\
\text { Coef. }(95 \% \mathrm{Cl})^{\mathbf{1}}\end{array}$ & $\begin{array}{c}\text { EQ-5D } 5 \mathrm{~L} \text { index score } \\
\text { Coef. }(95 \% \mathrm{Cl})^{\mathbf{2}}\end{array}$ \\
\hline 0 & Reference & Reference \\
1 & $-0.07(-0.09,-0.06)$ & $-0.06(-0.07,-0.05)$ \\
2 & $-0.12(-0.14,-0.10)$ & $-0.10(-0.12,-0.09)$ \\
3 & $-0.14(-0.17,-0.11)$ & $-0.12(-0.14,-0.11)$ \\
4 & $-0.16(-0.20,-0.11)$ & $-0.18(-0.21,-0.15)$ \\
$\geq 5$ & $-0.23(-0.27,-0.19)$ & $-0.22(-0.23,-0.20)$
\end{tabular}

Multimorbidity

\begin{tabular}{lcc} 
No & Reference & Reference \\
Yes & $-0.13(-0.15,-0.11)$ & $-0.12(-0.13,-0.11)$ \\
\hline
\end{tabular}

Difference in EQ-5D index score for the ${ }^{1} 2010$ and ${ }^{2} 2012$ Surveys of the HQCA; Adjusted (age, sex and household income).

\section{Comparing measurement properties of EQ-5D $3 \mathrm{~L}$ and EQ-5D 5L}

Missing values ranged from $0.1 \%$ for Self Care to $0.8 \%$ for Anxiety/Depression for the 3L version and $0.1 \%$ for Self Care to $0.6 \%$ for Anxiety/Depression for the 5L version. The proportion of respondents with at least one missing value in all dimensions was $1.3 \%$ for the $3 \mathrm{~L}$ version and $1.1 \%$ for the $5 \mathrm{~L}$ version, indicating good feasibility for both versions of the instrument.

There were a total of 384 unique health states observed using the $5 \mathrm{~L}$ ( $12.2 \%$ of 3125 possible) and 116 (47.7\% of 243 possible) using the $3 \mathrm{~L}$ version. A ceiling effect was observed among a greater proportion of respondents with the 3L, 2082/4946 (43.1\%) compared to 5L, 1536/4752 (32.3\%), with an absolute difference of $9.8 \%$. Among the most common chronic conditions, the highest difference was noted for high blood pressure (20.6\% versus $14.3 \%$ ) and high cholesterol (20.1\% versus $14.8 \%$ ) and similar for arthritis (6.9\% vs. $6.8 \%)$ and anxiety/depression (3.0\% for both).

EQ-5D instrument-dependent discriminatory power is represented by Shannon indices in Figure 1. Absolute discriminatory power (Shannon Index) was substantially higher in information richness in the $5 \mathrm{~L}$ classification system for all dimensions $\left(\mathrm{H}^{\prime}{ }_{5 \mathrm{~L}} / \mathrm{H}^{\prime}{ }_{3 \mathrm{~L}}\right)$ : Mobility $(0.80 /$ $0.50)$; Self-Care (0.24/0.16); Usual Activity (0.79/0.52); Pain/Discomfort (1.22/0.82), Anxiety/Depression (0.89/ $0.61)$ and overall $(0.87 / 0.59)$. Relative discriminatory power (Shannon Evenness index) that emphasizes the evenness of spread of responses across all levels by adjusting for the number of levels per instrument was slightly higher for all dimensions of $5 \mathrm{~L}\left(\mathrm{~J}_{5 \mathrm{~L}} / \mathrm{J}^{\prime}{ }_{3 \mathrm{~L}}\right)$ : Mobility $(0.09 / 0.06)$; Self-Care (0.03/0.02); Usual Activity (0.09/0.06); Pain/ Discomfort (0.14/0.10); Anxiety/Depression (0.11/0.07) and overall $(0.09 / 0.06)$. The absolute discriminatory power was higher on average with the $5 \mathrm{~L}$ (mean 0.79 versus 0.52 for $3 \mathrm{~L}$ ), and the relative discriminatory power was also reasonably higher for the $5 \mathrm{~L}$ (mean 0.09 versus 0.06 for $3 \mathrm{~L}$ ), confirming our hypothesis.

\section{Discussion}

The present study compares the measurement properties of EuroQol's EQ-5D 3L and the newer 5L in terms of their feasibility, ceiling effect and discriminatory power (Shannon indices) as well as the association between clinical characteristics and EQ index score. In our comparison of $3 \mathrm{~L}$ and $5 \mathrm{~L}$, both were comparable in terms of feasibility, but $5 \mathrm{~L}$ had lower ceiling effect and higher discriminatory power. Overall, multimorbidity was associated with clinically important reduction in index scores using both versions of the instrument, although it was notable that the decrements associated with specific conditions were different between the $3 \mathrm{~L}$ and $5 \mathrm{~L}$ version. 


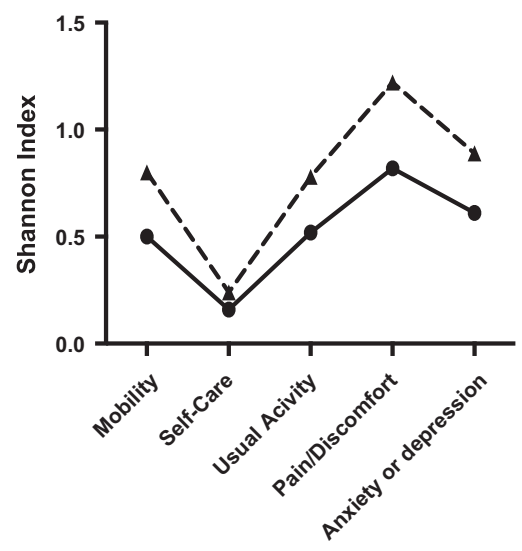

Five dimensions of the EQ-5D

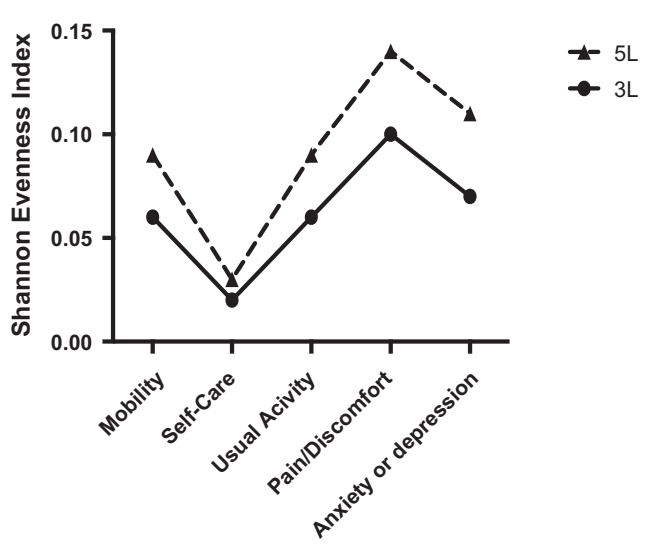

Five dimensions of the EQ-5D

Figure 1 Shannon index $\left(\mathrm{H}^{\prime}\right)$ and Shannon Evenness index $\left(\mathrm{J}^{\prime}\right)$ values for the $3 \mathrm{~L}$ and $5 \mathrm{~L}$.

This population-based study utilizes large samples from two consecutive survey cycles that are representative of the general population. Chronic pain was associated with the highest clinically important difference in HRQL using both versions of the EQ-5D instrument, consistent with previous findings $[3,20,21]$. However, the magnitude of this difference tended to be larger using the $3 \mathrm{~L}$ compared to the $5 \mathrm{~L}$ for some chronic conditions. The difference for individual conditions such as chronic pain and anxiety or depression may be due to different weights for specific dimensions associated with symptoms of these chronic conditions. That is, these conditions have the biggest weight on level 3 of the $3 \mathrm{~L}$. Likely related to the symptom of pain, the score difference associated with arthritis was also larger for the $3 \mathrm{~L}$ than $5 \mathrm{~L}$.

On the other hand, the associations between chronic conditions and index scores were similar for most chronic conditions, including multimorbidity using both versions of the EQ-5D. This similarity may be due to the fact that health profiles of $5 \mathrm{~L}$ are mapped unto the $3 \mathrm{~L}$ to derive its index score using an interim scoring " $E Q-5 D$ $5 L$ Crosswalk Index Value Calculator" [13]. That is, the utilities for $5 \mathrm{~L}$ are derived by first cross-matching its profiles to those of 3L. It is therefore expected that studied associations using index scores from both versions will not be significantly different. On the other hand, similar findings may in fact indicate the lack of difference in $3 \mathrm{~L}$ and $5 \mathrm{~L}$ using their derived utility scores. As a unique scoring system is being awaited for $5 \mathrm{~L}$, further studies will be required to show if differences occur in association between clinical characteristics and HRQL for both versions of the instrument.

The observation that $5 \mathrm{~L}$ had lower ceiling effect than the $3 \mathrm{~L}$ has been reported in other studies using patient populations [7-9]. The finding supports the original intent of increasing the levels in $5 \mathrm{~L}$, to capture differences in health states that are otherwise not captured by 3L. Moreover, the $5 \mathrm{~L}$ version of the EQ-5D descriptive system had a higher absolute discriminatory power than the $3 \mathrm{~L}$ version in all five dimensions. Also, the relative discriminatory power (Evenness index) was slightly better in the $5 \mathrm{~L}$ than the $3 \mathrm{~L}$ version. This measure indicates the evenness of spread of responses across levels of the instrument by adjusting for the number of levels. Thus, higher evenness scores in all $5 \mathrm{~L}$ dimensions indicate that the extra levels in the descriptive system were used efficiently. Our study findings indicate that the measurement properties of $5 \mathrm{~L}$ are better than $3 \mathrm{~L}$ in a general population sample. Further longitudinal analysis is needed to compare the sensitivity of both versions of the EQ-5D and their ability to detect change over time.

This study has a few limitations that are worth mentioning. Although the source population was the same in both survey cycles, the study samples for instrument comparison are not the same. The average number of chronic conditions was higher in the 2012 compared to 2010 survey cycle. This may be due to inherent differences in the study samples or under-reporting in the 2010 cycle, especially because of a skip pattern in the order of questions for identifying chronic conditions in that cycle. It is unclear to what extent this difference has on the comparison. It is unlikely to affect our comparison of specific conditions that have a higher prevalence, but may limit comparisons of total chronic conditions in the population. Furthermore, the results are consistent with findings from previous studies that are based on the same samples to validate both versions of the instrument [6-8]. Respondents' chronic conditions were self-reported. Because chronic conditions can be quite subtle, it may be confusing to differentiate between symptoms or minor ailments with more severe disease states. Moreover, some conditions such as chronic pain are subjective and may be difficult to 
define without assistance from a clinician or the use of standardized scales. The severity of chronic conditions, an important predictor of HRQL [2], was not accounted for in the present study. On the other hand, a unique property of our study is the large sample size derived from the general population, heightening the external validity of our study findings. Also, the study captured common chronic conditions in the general population, including the core chronic conditions recommended for inclusion in multimorbidity indices [22].

\section{Conclusions}

In this study, we found that the EQ-5D 5L showed better measurement properties, with lower ceiling effect and better discriminatory power than the $3 \mathrm{~L}$ version. Furthermore, while the association between overall multimorbidity and index scores is comparable using both versions of the EQ$5 \mathrm{D}$, the different versions suggest notable differences in HRQL burden for individual chronic conditions.

\section{Competing interests}

The authors declare that they have no competing interests.

\section{Authors' contributions}

ACB: conception and design, statistical analysis and interpretation of data, drafting manuscript, revision of manuscript ML: data acquisition, survey instrument and design, critical revision of manuscript TC: data acquisition, survey instrument and design, critical revision of manuscript JAJ: conception and design, data acquisition and interpretation of data, critical revision of manuscript. All authors read and approved the final manuscript.

\section{Author details}

${ }^{1}$ Department of Public Health Sciences, 2-040 Li Ka Shing Center for Health Research and Innovation, University of Alberta, Edmonton, Alberta, T6G 2E1, Canada. ${ }^{2}$ Health Quality Council of Alberta, Calgary, Alberta, Canada.

Received: 19 February 2014 Accepted: 9 May 2014

Published: 16 May 2014

\section{References}

1. Rabin R, de Charro F: EQ-5D: a measure of health status from the EuroQol Group. Ann Med 2001, 33:337-343.

2. Fortin M, Bravo G, Hudon C, Lapointe L, Almirall J, Dubois M-F, Vanasse A: Relationship between multimorbidity and health-related quality of life of patients in primary care. Qual Life Res 2006, 15:83-91.

3. Agborsangaya CB, Lau D, Lahtinen M, Cooke T, Johnson JA: Health-related quality of life and healthcare utilization in multimorbidity: results of a cross-sectional survey. Qual Life Res 2013, 22:791-799.

4. Pickard AS, De Leon MC, Kohlmann T, Cella D, Rosenbloom S: Psychometric comparison of the standard EQ-5D to a 5 level version in cancer patients. Med Care 2007, 45:259-263.

5. Pickard AS, Kohlmann T, Janssen MF, Bonsel G, Rosenbloom S, Cella D: Evaluating equivalency between response systems: application of the Rasch model to a 3-level and 5-level EQ-5D. Med Care 2007, 45:812-819.

6. Janssen MF, Birnie E, Haagsma JA, Bonsel GJ: Comparing the standard EQ-5D three-level system with a five-level version. Value Health 2008, 11:275-284.

7. Janssen MF, Pickard AS, Golicki D, Gudex C, Niewada M, Scalone L, Swinburn P, Busschbach J: Measurement properties of the EQ-5D-5L compared to the EQ-5D-3L across eight patient groups: a multi-country study. Qual Life Res 2013, 22(7):1717-1727.

8. Scalone L, Ciampichini R, Fagiuoli S, Gardini I, Fusco F, Gaeta L, Del Prete A, Cesana G, Mantovani LG: Comparing the performance of the standard EQ-5D $3 \mathrm{~L}$ with the new version EQ-5D 5L in patients with chronic hepatic diseases. Qual Life Res 2013, 22:1707-1716.
9. Kim SH, Kim HJ, Lee SI, Jo MW: Comparing the psychometric properties of the EQ-5D 3L and EQ-5D 5L in cancer patients in Korea. Qual Life Res 2012, 21:1065-1073.

10. HQCA: Satisfaction and Experience with Health Care Services: A Survey of Albertans. Calgary: Health Quality Council of Alberta; 2010.

11. Agborsangaya CB, Lau D, Lahtinen M, Cooke T, Johnson JA: Multimorbidity prevalence and patterns across socioeconomic determinants: a cross-sectional survey. BMC Public Health 2012, 12:201.

12. Shaw JW, Johnson JA, Coons SJ: US valuation of the EQ-5D health states: development and testing of the D1 valuation model. Med Care 2005, 43:203-220.

13. van Hout B, Janssen MF, Feng YS, Kohlmann T, Busschbach J, Golicki D, Lloyd A, Scalone L, Kind P, Pickard AS: Interim scoring for the EQ-5D 5L: mapping the EQ-5D 5L to EQ-5D 3L value sets. Value Health 2012, 15:708-715.

14. Kaplan RM: The minimally clinically important difference in generic utility-based measures. COPD 2005, 2:91-97.

15. Shannon CE: The mathematical theory of communication. 1963. MD Comput 1997, 14:306-317.

16. Rao GS, Hamid Z, Rao JS: The information content of DNA and evolution. J Theor Biol 1979, 81:803-807.

17. Dahl FA, Østerås N: Quantifying information content in survey data by entropy. Entropy 2010, 2:161-163.

18. Janssen MF, Birnie E, Bonsel GJ: Quantification of the level descriptors for the standard EQ-5D three-level system and a five-level version according to two methods. Qual Life Res 2008, 17:463-473.

19. Polinder S, Haagsma JA, Bonsel G, Essink-Bot ML, Toet H, van Beeck EF: The measurement of long-term health-related quality of life after injury: comparison of EQ-5D and the health utilities index. Inj Prev 2010, 16:147-153.

20. Lame IE, Peters ML, Vlaeyen JW, Kleef M, Patijn J: Quality of life in chronic pain is more associated with beliefs about pain, than with pain intensity. Eur J Pain 2005, 9:15-24.

21. Horng YS, Hwang YH, Wu HC, Liang HW, Mhe YJ, Twu FC, Wang JD: Predicting health-related quality of life in patients with low back pain. Spine 2005, 30:551-555.

22. Diederichs C, Berger K, Bartels DB: The measurement of multiple chronic diseases-a systematic review on existing multimorbidity indices. J Gerontol A Biol Sci Med Sci 2011, 66:301-311.

doi:10.1186/1477-7525-12-74

Cite this article as: Agborsangaya et al:: Comparing the EQ-5D 3L and $5 \mathrm{~L}$ : measurement properties and association with chronic conditions and multimorbidity in the general population. Health and Quality of Life Outcomes 2014 12:74.

\section{Submit your next manuscript to BioMed Central and take full advantage of:}

- Convenient online submission

- Thorough peer review

- No space constraints or color figure charges

- Immediate publication on acceptance

- Inclusion in PubMed, CAS, Scopus and Google Scholar

- Research which is freely available for redistribution 